\title{
The impact of large scale ionospheric structure on radio occultation retrievals
}

\author{
A. J. Mannucci, C. O. Ao, X. Pi, and B. A. Iijima \\ Jet Propulsion Laboratory, California Institute Of Technology, Jet Propulsion Laboratory, MS 138-308, \\ 4800 Oak Grove Drive, Pasadena, CA 91109, USA
}

Received: 26 February 2011 - Published in Atmos. Meas. Tech. Discuss.: 4 May 2011

Revised: 24 October 2011 - Accepted: 10 November 2011 - Published: 22 December 2011

\begin{abstract}
We study the impact of large-scale ionospheric structure on the accuracy of radio occultation (RO) retrievals. We use a climatological model of the ionosphere as well as an ionospheric data assimilation model to compare quiet and geomagnetically disturbed conditions. The presence of ionospheric electron density gradients during disturbed conditions increases the physical separation of the two GPS frequencies as the GPS signal traverses the ionosphere and atmosphere. We analyze this effect in detail using ray-tracing and a full geophysical retrieval system. During quiet conditions, our results are similar to previously published studies. The impact of a major ionospheric storm is analyzed using data from the 30 October 2003 "Halloween" superstorm period. At $40 \mathrm{~km}$ altitude, the refractivity bias under disturbed conditions is approximately three times larger than quiet time. These results suggest the need for ionospheric monitoring as part of an RO-based climate observation strategy. We find that even during quiet conditions, the magnitude of retrieval bias depends critically on assumed ionospheric electron density structure, which may explain variations in previously published bias estimates that use a variety of assumptions regarding large scale ionospheric structure. We quantify the impact of spacecraft orbit altitude on the magnitude of bending angle and retrieval error. Satellites in higher altitude orbits $(700+\mathrm{km})$ tend to have lower residual biases due to the tendency of the residual bending to cancel between the top and bottomside ionosphere. Another factor affecting accuracy is the commonly-used assumption that refractive index is unity at the receiver. We conclude with remarks on the implications of this study for long-term climate monitoring using RO.
\end{abstract}

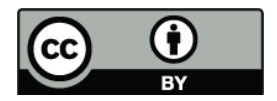

Correspondence to: A. J. Mannucci (tony.mannucci@jpl.nasa.gov)

\section{Introduction}

The Earth's global climate is a subject of intense scientific and practical interest. The radio occultation remote sensing technique offers the possibility of precise and accurate atmospheric soundings that are well-suited for observing decadalscale climate change. A particularly favorable aspect of radio occultation is that atmospheric parameters are retrieved based on a measurement of radio signal phase and phase rate. The fundamental measurement is therefore derived from signal timing, which is calibrated on-orbit to standards traceable to fundamental SI units.

Radio occultation uses a physically based retrieval scheme (Kursinski et al., 1996; Rocken et al., 1997) that permits detailed analyses of sources of measurement bias. Such analyses are needed to ensure that measurement accuracy is absolutely calibrated to the standard SI units. Detailed error analyses have been published (Kursinski et al., 1997; Hajj et al., 2002; Kuo et al., 2004; Hajj et al., 2004; Steiner and Kirchengast, 2005) that analyze nearly all of the known error sources. For monitoring decadal-scale climate change, measurement bias should be less than $\sim 0.1 \mathrm{~K}$ (Ohring et al., 2005; Goody et al., 1998; Steiner et al., 2001), which motivates a reexamination of these past analyses that were focused initially on establishing precision of individual soundings at the level of $\sim 1 \mathrm{~K}$ (Kursinski et al., 1997).

Recent studies that compare RO retrievals to other in-situ and remote methods, and simulation studies that consider various sources of measurement error, tend to confirm the high accuracy of RO above the lower troposphere (Hajj et al., 2004; Kuo et al., 2005; Schreiner et al., 2007; Sun et al., 2010; Xu et al., 2009; He et al., 2009; Hayashi et al., 2009). The GPS RO technique achieves high accuracy by using "self-calibration": data acquired during the measurement also serves to calibrate the observations. An example of self-calibration is the simultaneous tracking of a GPS "calibration satellite" while the occulting satellite is tracked. The

Published by Copernicus Publications on behalf of the European Geosciences Union. 
calibration satellite is a GPS satellite in view above the local spacecraft horizon. The additional satellite provides timing data that is combined with the occulting satellite data to remove receiver clock error from the retrieval. Thus, the retrieved atmospheric properties are not susceptible to receiver clock error.

Another form of self-calibration is used to reduce timing errors due to the Earth's ionosphere and plasmasphere, a medium of tenuous plasma at altitudes between $\sim 90 \mathrm{~km}$ and the GPS satellites orbiting at $20200 \mathrm{~km}$ (hereafter we use the term ionosphere exclusively to imply both ionosphere and plasmasphere). The ionospheric refractive index introduces delay and delay rate to the GPS signal. Calibrating ionospheric delay is accomplished by tracking the two GPS signal transmission frequencies: L1 $(1.575 \mathrm{MHz})$ and L2 (1.228 MHz). The delay difference between the two frequencies is caused by the ionosphere, which is calculated to high accuracy using well-understood physical principles and formulas that describe the refractive index dispersion of the ionospheric plasma. In contrast, the frequency dispersion of the neutral troposphere and stratosphere refractive index is negligible at GPS frequencies. Knowledge of the differential delay between L1 and L2 frequencies is used to calibrate precisely the ionospheric contribution.

Residual ionospheric calibration errors remaining after applying the dual-frequency correction are not negligible for climate applications. The calibration is degraded by two factors. First, the L1 and L2 signal raypath trajectories through the ionosphere are not identical. The dual-frequency correction is incomplete if raypath separation is not accounted for. Fully accounting for raypath separation requires knowledge of electron density gradients along the raypath. Second, the refractive index gradient at each frequency depends on the magnetic field along the raypath, which is not accounted for in standard "first-order" calculations of ionospheric dispersion (so-called "higher-order" ionospheric effects). See Syndergaard (2000), Vergados and Pagiatakis (2010) and Bassiri and Hajj (1993).

In this paper, we present a detailed analysis of residual ionospheric calibration error. We address the impact of raypath separation between the two GPS frequencies, caused by large-scale electron density gradient structures in the ionosphere. We perform detailed ray-tracing calculations to analyze the occulting raypath geometries in realistic electron density structures using realistic transmitter-receiver geometries. The ionospheric electron density fields are obtained from global climatological and data assimilation models of the ionosphere. Data assimilation is needed to characterize the ionosphere under geomagnetically disturbed conditions. The analysis in this paper represents the first time that ionospheric data assimilation modeling is applied to a study of ionospheric calibration accuracy for RO. In the next section, we discuss the nature of the residual error in more detail. In Sect. 3 we describe the analysis method. Results and their discussion are treated in Sects. 4 and 5.

\section{Origins of ionospheric residual error}

The Earth's ionosphere is an ionized atmospheric medium containing a significant number of free electrons primarily in the altitude range $\sim 90-1200 \mathrm{~km}$. At the transmission frequencies of GPS, the refractive index (polarizability) of free electrons is far larger than that of neutral gas per unit mass. The refractive index of the daytime ionosphere at $\sim 300 \mathrm{~km}$ altitude is comparable to the stratospheric refractive index at about $\sim 20-30 \mathrm{~km}$ altitude, although the densities of these two media differ by more than 10 orders of magnitude. Retrieving atmospheric properties requires calibration of ionospheric effects on the signal, particularly for climate benchmark applications applied to the upper troposphere and stratosphere. In the mid-to-lower troposphere, residual refractivity or temperature errors due to ionosphere are less than $0.01 \%$ (Kursinski et al., 1997).

Accommodating ionospheric residual bias from a climate perspective is achieved by setting reliable upper bounds on that bias, and reducing the bias by algorithmic and data processing approaches if possible. To achieve SI-traceable accuracy in the presence of uncertain electron density structure, robust upper bounds on residual error are needed so that all realistic ionospheric density configurations will result in residual errors less than the bound. We expect that very severe ionospheric storms that occur a few times per solar cycle may violate the upper bound. However, their impact on climate averages is easily removed by monitoring ionospheric disturbance levels with widely available resources such as global GPS receiver networks.

Setting an upper bound on residual bias is achievable because of the physical nature of the RO retrieval process. Using physics-based simulation, we can calculate precisely the error in the atmospheric retrieval at a given altitude produced by a given electron density distribution in the ionosphere. Taking into account the possible range of electron density distributions leads to realistic upper bounds on the residual error. Implementing this approach is not trivial, and has not yet been achieved by the RO research community. This approach is realistic for large-scale ionospheric structures of the kind analyzed here, that are captured in climatological and data assimilation models of the ionosphere. A different approach is needed for E-region structures that may bias the retrievals, a subject that will be treated in future work.

Small-scale irregularities $(10 \mathrm{~s} \mathrm{~km})$ and the presence of E-region electron density peaks $(\sim 120 \mathrm{~km}$ altitude) are not considered in this paper. The impact of these smaller scale structures can be significant (Syndergaard, 2000; Hoque and Jakowski, 2010) but have quite different climatological characteristics than large scale structure and will be more difficult to model in a deterministic fashion. Therefore, for climate applications, the impact of small-scale structures must be treated statistically. The signature of these structures will be discernable in the data as fluctuations in the signal amplitude, phase and measured total electron content (TEC). A 
future study is needed to determine methods for bounding errors due to small scale variability.

In the next sections, we describe the standard GPS approach to calibrating ionospheric delays, and the causes of residual calibration bias. We then show the results of our study to quantify residual bias using simulation. Our analysis should be useful to establishing SI-traceability in the presence of retrieval bias due to the ionosphere, at least for effects caused by large-scale ionospheric structure (see Fox et al., 2011 and references therein for a discussion of accuracy and SI-traceability).

\subsection{Dual frequency ionospheric correction}

Ionospheric correction for GPS measurements is applied using the GPS data itself, by forming linear combinations of the carrier phase information at both transmission frequencies. Geophysical observables derived from GPS radio occultation depend fundamentally on the measured Doppler shift at each GPS frequency, caused by refractive index variations within the neutral atmosphere and ionosphere. Differences in the Doppler shift between the two GPS frequencies are due to effects of the ionosphere. The physical basis by which Doppler shift varies with frequency is well understood. Algorithms have been developed that use the measurements at both frequencies to create a new observable that is nearly free of ionospheric effects, thus creating an observable that depends only on the atmospheric refractive index (Hajj et al., 2002). The algorithms use the fact that, to first order, the phase delay incurred by the ionosphere is proportional to the inverse square of the signal frequency $\left(1 / f^{2}\right)$.

Residual ionospheric error occurs because the phase delay is not exactly proportional to $1 / f^{2}$. Two primary factors cause deviation from the $1 / f^{2}$ dependence. Higher order ionospheric effects due to the geomagnetic field introduce $1 / f^{3}$ (cubic) terms in the phase delay that depend on the geomagnetic field strength and electron density distribution along the raypath. More significantly, spatial gradients of electron density cause the L1 and L2 raypaths to separate and sample different electron density distributions (Syndergaard, 2000; Ladreiter and Kirchengast, 1996; Kursinski et al., 1997; Gorbunov et al., 1996). The net effect is that the ionospheric contribution to phase delay does not vary exactly as $1 / f^{2}$. Deviation from $1 / f^{2}$ behavior depends in detail on the electron density structure of the ionosphere and the degree of separation along the occulting raypaths. Therefore, the magnitude of residual error varies with each occultation because of ionospheric variability or "weather".

In the following paragraphs we describe an approach widely used to apply the ionospheric correction to radio occultation data (Hajj et al., 2002). This approach is based on a procedure first suggested by Vorobév and Krasil'nikova (1993). The dual frequency correction is applied to the bending angles at the L1 and L2 frequencies, interpolated to a common impact parameter, not to the phase delays themselves. (Bending angle is a by-product of the measured Doppler shift using geometrical considerations; see Hajj et al., 2002). The impact parameter is the asymptotic distance of the rays from Earth's center as they leave the atmosphere (see Hajj et al., 2002 for a definition). The bending angle approach largely (but not completely) compensates for the separation of $\mathrm{L} 1$ and $\mathrm{L} 2$ raypaths, and provides a more accurate correction than applying the correction to the measured GPS phase delays. The following linear combination of L1 and L2 bending angles approximates the bending angle of the neutral atmosphere free of ionospheric effects:

$\alpha_{\text {neut }}\left(a_{0}\right)=C_{1} \alpha_{1}\left(a_{0}\right)-C_{2} \alpha_{2}\left(a_{0}\right)$

where $\alpha_{1}\left(a_{0}\right)$ and $\alpha_{2}\left(a_{0}\right)$ are the bending angles at the L1 and L2 frequencies, respectively, at impact parameter $a_{0}$. The constants $C_{1}$ and $C_{2}$ are functions of the two GPS frequencies $f_{1}$ and $f_{2}: C_{1}=F_{1}^{2} /\left(f_{1}^{2}-f_{1}^{2}\right)=2.545728$, and $C_{2}=f_{2}^{2} /\left(f_{1}^{2}-f_{2}^{2}\right)=1.545728$. Piecewise cubic interpolation of the bending angle versus impact parameter at each frequency is used to estimate the bending angle at the common impact parameter $a_{0}$. To reduce bending angle noise, an algebraic manipulation of this equation is formed as follows, using the fact that $C_{2}=\left(C_{1}-1\right)$ :

$\alpha_{\text {neut }}\left(a_{0}\right)=\alpha_{1}\left(a_{0}\right)+(C 1-1)\left(\bar{\alpha}_{1}\left(a_{0}\right)-\bar{\alpha}_{2}\left(a_{0}\right)\right)$

where $\bar{\alpha}_{1}\left(a_{0}\right)$ and $\bar{\alpha}_{2}\left(a_{0}\right)$ are time-smoothed versions of the bending angle time series at each frequency. Typically, the smoothing occurs over intervals of $\sim 2 \mathrm{~s}$, whereas the high rate bending angle $\alpha_{1}\left(a_{0}\right)$ is computed approximately every $1 / 2 \mathrm{~s}$ based on the size of the Fresnel diameter (Hajj et al., 2002). The overall noise reduction compared to using completely unsmoothed angles is approximately a factor of 7. See also Sokolovskiy et al. (2009) for optimized filtering approaches.

The constants $C_{1}$ and $C_{2}$ are the same as those used in the ionospheric correction formula for phase delay and delay rate. The correction in Eq. (1) works very well if there is a linear relationship between bending angle and phase delay rate. Raypath separation effects are reduced by interpolating to a common impact parameter $a_{0}$ (Ladreiter and Kirchengast, 1996). However, the ionospheric error is not eliminated by this interpolation when the ionosphere is not spherically symmetric. Non-linearity in the relationship between bending angle and phase delay also contributes to ionospheric residual error (Ladreiter and Kirchengast, 1996; Syndergaard, 2000; Gorbunov, 1996).

At altitudes below $\sim 10 \mathrm{~km}$, due to atmospheric defocusing and reduction of signal amplitude, the L2 signal is often too weak for robust tracking (Hajj et al., 2002; Kuo et al., 2004; Mannucci et al., 2006). At such altitudes, Eq. (2) cannot be applied. In that case, the "smoothed" ionospheric correction terms in Eq. (2) are extrapolated downward from higher altitudes to continue the ionospheric correction. The altitude range of the extrapolation is typically below $8-12 \mathrm{~km}$ as the 
second frequency is often lost at about $12 \mathrm{~km}$ altitude. A simulation study by Mannucci et al. (2006) suggests extrapolation may cause refractivity errors of $\sim 0.05 \%$ near the upper altitude range where the $\mathrm{L} 2$ loss first occurs.

The ionospheric calibration approach represented in Eq. (1) is used in the simulated refractivity retrievals presented in this study. We also analyze bending angles directly for the L1 and L2 frequencies, and compute the residual bending angle after interpolation to a common impact parameter.

\subsection{Residual ionospheric error: L1/L2 path separation and higher-order effects}

The most significant source of ionospheric bias is that refractive index (electron density) gradients along the raypath cause the L1 and L2 signals to follow different paths in the ionosphere (Hoque and Jakowski, 2010); a dual-frequency correction of the form Eq. (1) leads to residual errors if raypath separation affects the corrected bending angle. The full Appleton-Hartree formula for refractive index of the extraordinary ray (e.g. Davies, 1990; Bassiri and Hajj, 1993; Hoque and Jakowski, 2010) is used in our simulations, appropriate for the right-hand circularly polarized GPS transmission. A realistic representation of the Earth's magnetic field is used based on the IGRF model (see IAGA, 2003).

\section{Approach}

The propagation path of an electromagnetic wave through a medium such as the atmosphere or ionosphere is determined by the refractive index variations in the vicinity of the path. The path deviates from straight-line propagation due to spatial gradients in the refractive index near the path.

Ray-tracing determines the trajectories of the L1 and L2 raypaths as they travel from satellite transmitter to the receiver (Born and Wolf, 1980; Budden, 1985). A ray-tracing algorithm could in principle be used to determine the dependence of bending angle on impact parameter, as is needed for Eqs. (1) and (2). Ray-tracing is not typically used in the retrieval process because it is far more computationally intensive than traditional methods.

We perform detailed ray-tracing studies through representative ionospheres to study the impact of ionospheric structure on RO retrievals. Ray-tracing permits us to examine how properties of ionospheric structure impact the retrieval accuracy. For example, our study demonstrates that orbit altitude plays a role in the magnitude of residual ionospheric errors. We find that vertical electron density gradients in the topide ionosphere partially cancel the effect of gradients in the bottomside. Ray-tracing is used in Syndergaard et al. (2000) to validate the theoretical treatment of ionospheric residual found in that work, and is used by Hoque and Jakowski (2010) to determine how higher-order ionospheric refraction terms affect measurements of total electron content. In our calculations, the refractive index is calculated for the extraordinary ray, taking into account the orientation of the Earth's magnetic field with respect to the raypath direction.

Representative ionospheric electron density distributions are derived from two sources for this study: the International Reference Ionosphere (IRI) (Bilitza and Reinisch, 2008) and the Global Assimilative Ionosphere Model (GAIM) developed at JPL and the University of Southern California (Wang et al., 2004). IRI is a widely used climatological ionosphere model that provides values of electron density at any worldwide location specified by altitude, latitude and longitude. IRI uses as input the F10.7 index, as a proxy for solar radiation in the extreme ultraviolet and soft X-ray bands. The index is based on measurements of solar emittance at $10.7 \mathrm{~cm}$ wavelength. IRI electron density distributions represent how electron density varies with solar cycle and local time, two important factors that determine ionospheric variability. Studies using IRI and other models suggest that residual ionospheric effects are largest near solar maximum daytime, but are negligible $(<0.05 \mathrm{~K})$ at nighttime and during solar minimum, from altitudes $25 \mathrm{~km}$ downward (Kursinski et al., 1997).

We use the Global Assimilative Ionosphere Model (GAIM) as an another source of electron density distribution. GAIM is used to analyze cases that deviate from average climatological conditions. GAIM is a space weather prediction model patterned after numerical weather prediction models for the troposphere and stratosphere. GAIM uses sources of global ionospheric data such as from GPS measurements and other systems to augment climatological or physics-based representations. Techniques such as GAIM have already shown great promise in improving upon climatology to produce three-dimensional maps of ionospheric electron density on global scales. We use GAIM assimilation runs during an extreme ionospheric space weather event in 2003 to analyze residual ionospheric errors under extremely unfavorable conditions. Input data for these runs is based on ground-based GPS receivers distributed globally, measuring total electron content above the receiver locations (Mannucci et al., 1998).

Previous research (Kursinski et al., 1997; Steiner et al., 2001) has shown that residual ionospheric bias can approach $0.3 \mathrm{~K}$ at altitudes between $25-35 \mathrm{~km}$ during daytime solar maximum conditions. Recent research and theoretical work (Syndergaard, 2000) indicates that residual bias increases with electron density magnitudes, which are often controlled by the radiance level of the Sun at EUV and shorter wavelengths. Therefore, residual bias depends on the electron density magnitudes assumed. These magnitudes in turn depend on the assumed solar radiation environment. For a given solar radiation environment and quiet geomagnetic conditions, electron density magnitudes can often deviate from climatological behavior by a factor of two (Brown et 


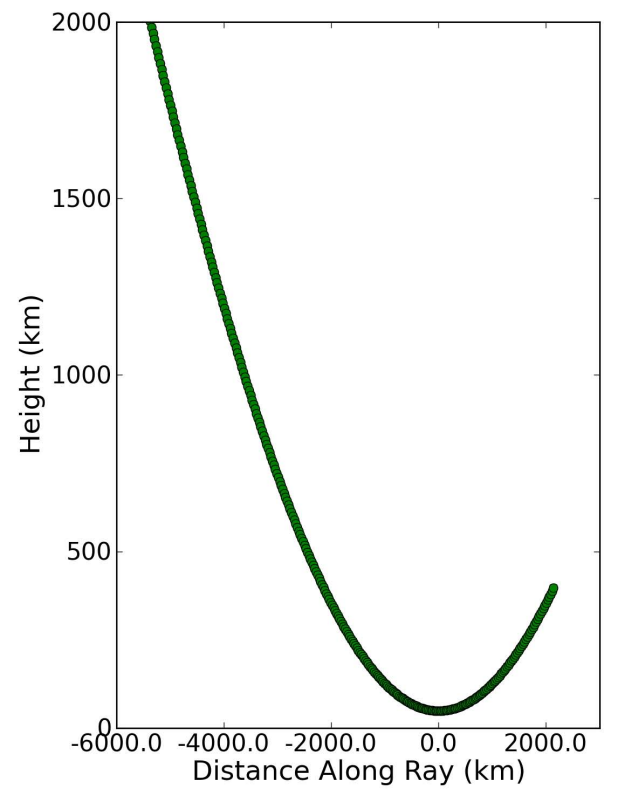

Fig. 1. The altitude of the simulated ray versus distance along the ray, starting at $2000 \mathrm{~km}$ altitude. Zero distance is at the ray tangent point. The receiver is on-board CHAMP (400 km altitude orbit).

al., 1991). Variations of electron density near solar maximum daytime conditions must be accounted for to set reliable upper bounds on the level of ionospheric residual bias in retrievals. Ionospheric storms, which are byproducts of geomagnetic activity, can increase ionospheric residuals significantly compared to quiet geomagnetic conditions. The residual bias increases for two reasons that often occur simultaneously during storms: overall electron density values increase as do their spatial gradients. According to propagation physics (Born and Wolf, 1980), the raypaths followed at the L1 and L2 frequencies depend on refractive index magnitudes and spatial gradients. Separation of raypaths between L1 and L2 generally increases under both density increases and gradient increases for fixed density. Ionospheric storms also undergo "negative" phases, resulting in a significant decrease of electron density relative to quiet conditions. During these periods, we expect the ionospheric residual to decrease, so we do not explicitly focus on this phase.

In the next section we discuss analysis of the ray-tracing results under representative quiet and disturbed conditions. Our emphasis is on daytime solar maximum conditions when the ionospheric residuals are largest. Simulations for nighttime and solar minimum are not considered. We show in detail which parts of the topside and bottomside ionospheric electron density profile are cause for greatest raypath separation, not considering the impact of pronounced E-layers which are important also.

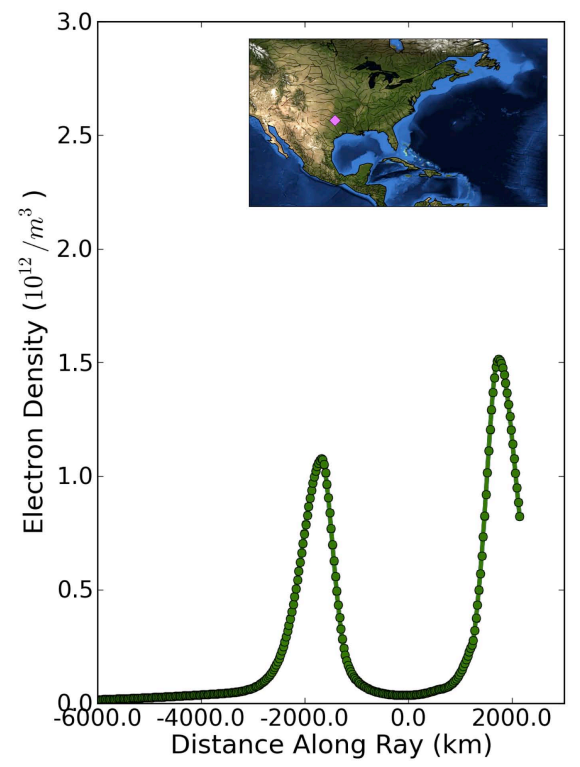

Fig. 2. Electron density versus distance along the simulated raypath. The curves for L1 and L2 frequencies overlay nearly exactly on this scale. The location of the occultation tangent point is shown in the inset.

\section{Results}

A representative occultation raypath has been selected for detailed study in these simulation experiments. The minimum altitude of the raypath (tangent point) is $60 \mathrm{~km}$. At such high altitudes, ionospheric residuals will produce a significant impact on retrieval error. The goal of achieving temperature biases less than $0.1 \mathrm{~K}$ is unrealistic at $60 \mathrm{~km}$ and higher altitudes, limiting the possibility of using radio occultation in applications where SI-traceability is desired at such high altitudes. At lower altitudes, atmospheric bending increases and the impact of ionospheric residuals is less.

We have used the end points of the ray-path from an actual occultation acquired by the CHAMP satellite (Wickert et al., 2001) on 30 October 2003. The altitude of the ray versus distance from the tangent point (ray perigee) is shown in Fig. 1. The location of the raypath tangent point is shown as the inset in Fig. 2. A severe geomagnetic storm was in progress on 30 October 2003. Very large ionospheric total electron content and electron density spatial gradients were detected by a ground-based GPS receiver network in the vicinity of the occultation ray-path (for details, see Mannucci et al., 2005). This particular occultation geometry was selected to coincide with extreme ionospheric conditions as represented by the GAIM.

In the following we provide detailed simulation results for L1/L2 ray-path separation. We show results using the IRI-95 climatological model and using GAIM updated with TEC measurements from the ground to represent storm-time 


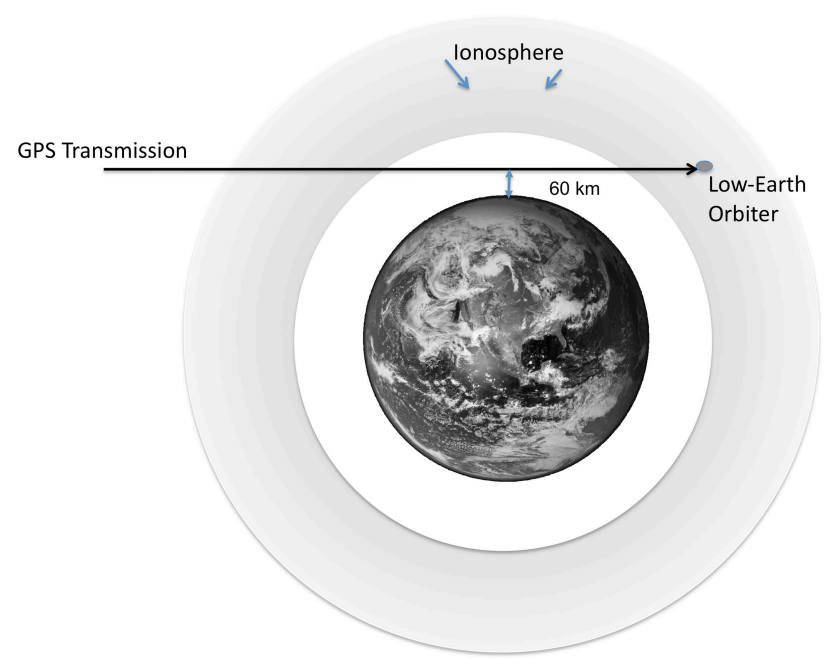

Fig. 3. Schematic representation of the simulated raypath as it enters, exits then re-enters the ionosphere before meeting with the receiver.

conditions. The latter produces much larger gradients than is present in the IRI-95 climatology.

\subsection{Results using the International Reference Ionosphere}

The IRI-95 model was run for 30 October 2003. The F10.7 solar flux index on that day was 267 (adjusted value), a very high value exceeding by $\sim 48 \%$ the average F10.7 value during solar maximum years 2000-2001. The electron density encountered along the raypath is shown in Fig. 2. The twopeak structure is a result of twice traversing the ionospheric "annulus", as shown schematically in Fig. 3. In Fig. 2, the electron densities for the L1 and L2 paths differ imperceptibly due to the slightly different raypaths and do not appear in this figure. The two electron density peak values occur when the raypath is traversing altitudes 260 and $250 \mathrm{~km}$, near the altitudes of peak electron density in the model (see right panel, Fig. 12, for vertical electron density profiles from the IRI-95 output near the tangent point). Electron densities were linearly extrapolated downward from altitudes of $100 \mathrm{~km}$ since the model run cuts off abruptly at that altitude. For this study, we determine the end points of the ray-path from an actual CHAMP orbit when the satellite altitude was $400 \mathrm{~km}$. We will show later that the altitude of the receiver has an impact on the magnitude of the ionospheric residual bias.

The accumulated bending angle along the raypath for the $\mathrm{L} 1$ and L2 frequencies is shown in Fig. 4. The bending angle is with respect to the direction that the raypath leaves the transmitter. The angle differs significantly between the two frequencies because of the frequency-dependent refractive index according to the $1 / f^{2}$ law. The accumulated

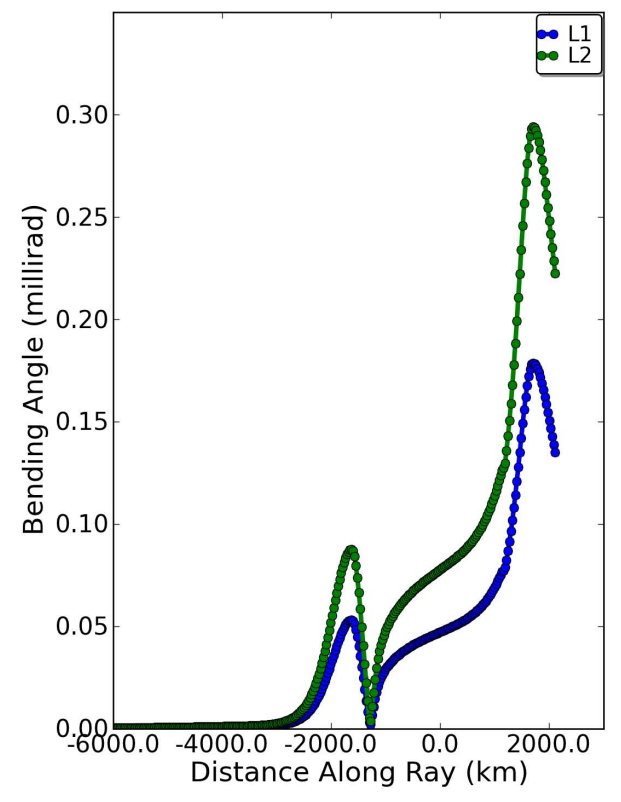

Fig. 4. Bending angle along the ray for the $\mathrm{L} 1$ and $\mathrm{L} 2$ frequencies, assuming IRI electron density distribution extrapolated linearly to zero below $100 \mathrm{~km}$. Bending angle is relative to the direction that the ray leaves the transmitter.

bending angle follows a similar overall pattern for the two frequencies. Several features are notable. Near $-1300 \mathrm{~km}$, the accumulated bending angle approaches very low values. At this point along the raypath, the bending due to the topside ionosphere is nearly compensated for by the oppositelydirected bending in the bottomside, yielding a small net bending value. Shortly after this minimum, the raypath drops below $100 \mathrm{~km}$, effectively exiting the ionosphere. The bending angle increases slowly due to the residual density from the extrapolated IRI profile. The raypath reenters the ionosphere on the bottom side at $\sim 1000 \mathrm{~km}$ distance, at which time bending angle increases rapidly again. The electron density peak is reached at $\sim 1900 \mathrm{~km}$ distance, at which time the bending angle direction begins to reverse again. The net bending angle at the receiver is $\sim 0.13$ millirad for the L1 frequency, reached at $\sim 2200 \mathrm{~km}$.

The retrieval of geophysical parameters depends on the accumulated bending angle at the location of the GPS receiver, which is generally orbiting at a radius within the ionosphere. Since the accumulated bending angle does not increase monotonically, certain altitudes for the GPS receiver are more favorable for obtaining low residual biases. For this example, altitudes near $195 \mathrm{~km}$ yield zero bending angle due to cancellation from the topside and bottomside ionosphere. The altitude of this complete cancellation will vary with electron density distribution. More generally (and realistically), higher altitudes above the F2 peak density (above $500 \mathrm{~km}$ ) will result in more cancellation of the accumulated bending, as can be seen by the bending angle trends near the end of the 


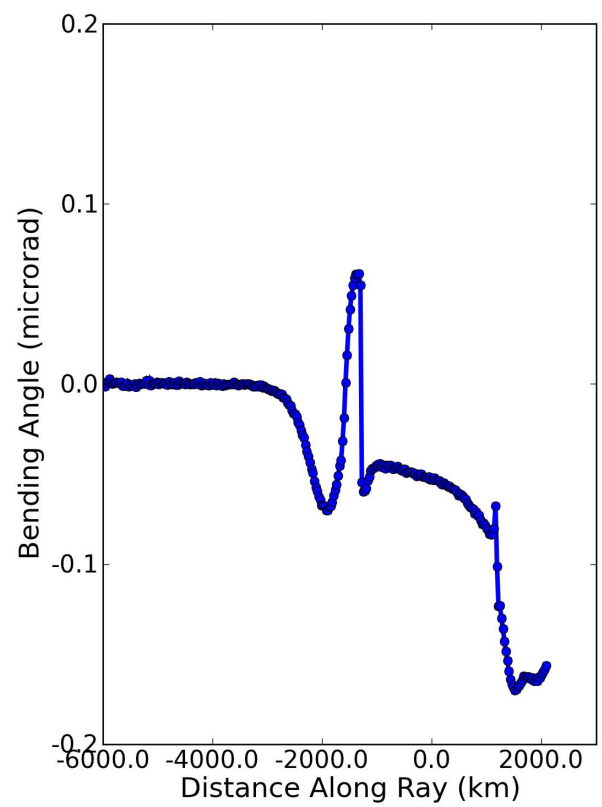

Fig. 5. Residual bending angle after the dual-frequency correction is applied, IRI case.

raypath in Fig. 4. The reduced residual ionosphere at higher altitudes is further discussed in Sect. 4.3.

The linear combination of bending angles at the L1 and L2 frequencies (Eq. 1) produces an estimate of bending caused solely by the neutral atmosphere. Electron density gradients along the L1 and L2 raypaths result in imperfect cancellation of ionospheric effects when this formula is applied. In Fig. 5, we plot the residual bending angle calculated from our ray-tracing simulation, assuming the dual-frequency correction is applied. Deviations from zero in Fig. 5 are a measure of ionospheric residual due to raypath separation and higher-order ionospheric effects, either positive or negative depending on whether the dual-frequency correction underestimates or overestimates the actual bending angle, respectively. The residual bending changes rapidly as the raypath enters and exits the first ionospheric traversal. On exiting the ionosphere, the residual bending remains nearly constant with a small bias of $\sim-3 \times 10^{-8}$ radians. The structure of the ionosphere results in a fortuitous cancellation of bending angle from the top and bottomsides, as discussed before. Bending angle residual magnitude again begins to increase as the raypath enters the bottomside for the second time $(\sim 1000 \mathrm{~km})$. For this second traversal, cancellation in the topside barely occurs since the satellite is orbiting near the altitude of peak density. At the receiver, the residual bending is $\sim-6 \times 10^{-8}$ radians, which is the value relevant to retrieval error. If the receiver were at higher altitude, bending cancellation would occur similarly to what occurs in the first ionospheric traversal, reducing the overall impact of the ionosphere on the retrieval. The impact of satellite altitude

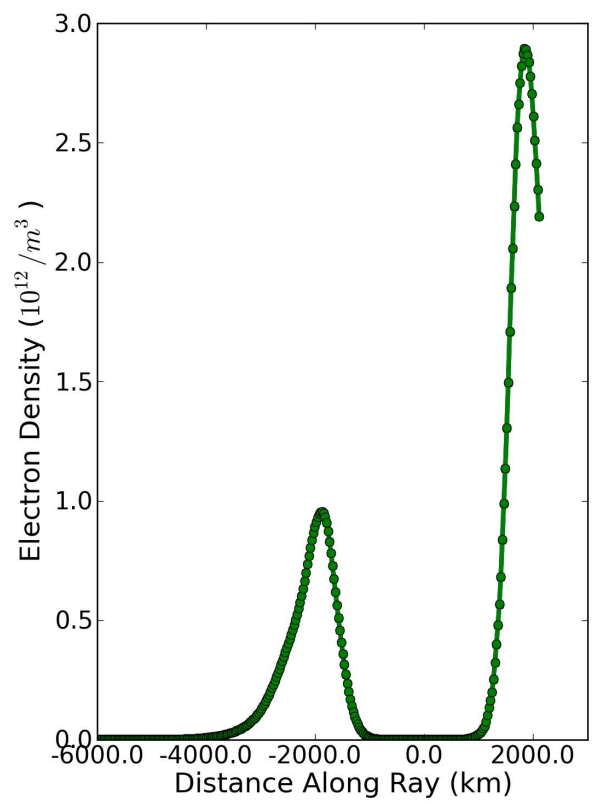

Fig. 6. Electron density versus distance along the ray for the simulated raypath assuming the GAIM electron density distribution (compare to Fig. 2).

is discussed later for a low-Earth orbiter (LEO) at COSMIC altitudes ( $780 \mathrm{~km}$ versus $400 \mathrm{~km}$ for CHAMP).

\subsection{Global assimilative ionosphere model - major ionospheric storm}

We use the GAIM to assess the impact of significant electron density gradients that occur during geomagnetic storms, conditions that are not generally captured by the IRI. Plots analogous to those just described for IRI are Figs. 6-8. The transmitter-receiver locations are identical in the two cases. The GAIM assimilates total electron content (TEC) data from the global network of Global Positioning System (GPS) ground receivers and thus captures, at least approximately, horizontal electron gradients and magnitudes that occurred during the storm. The GPS TEC data captures storm conditions that occurred on 30 October 2003. The storm is characterized by large TEC daytime values, enhanced spatial gradients and vertical "uplift" of electron density as reported in Mannucci et al. (2005).

The GAIM results confirm that electron densities reach larger magnitudes compared to quiet time. Electron densities differ significantly between the two traversals of the transionospheric propagation (Fig. 6). Peak electron density in the entrance lobe of the ionosphere is slightly lower than IRI in the storm-time case represented by GAIM. In the second lobe, the peak electron density is a factor of 1.75 larger in the storm case compared to IRI. Bending angle (Fig. 7) for the storm case is significantly larger in the second lobe compared to the first lobe. 


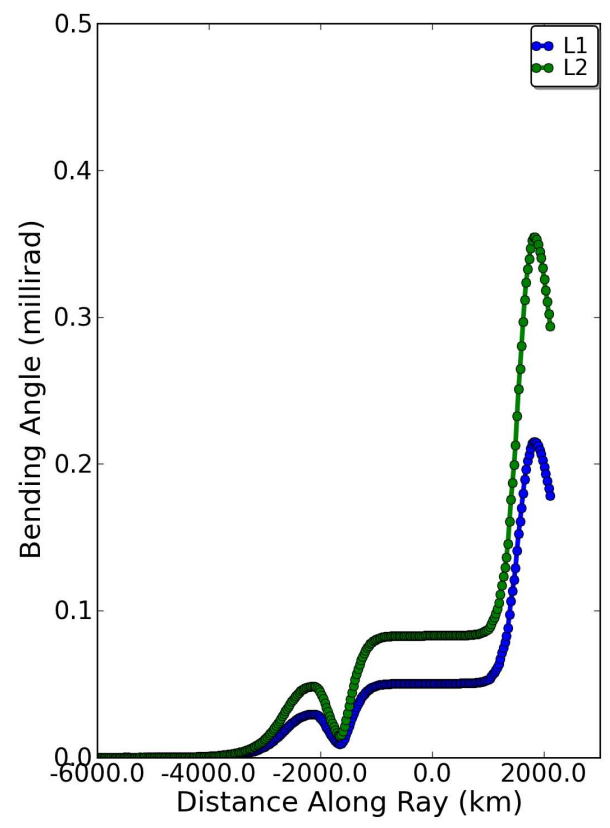

Fig. 7. Bending angle along the ray for the L1 and L2 frequencies, assuming GAIM electron density distribution. Compare to Fig. 4.

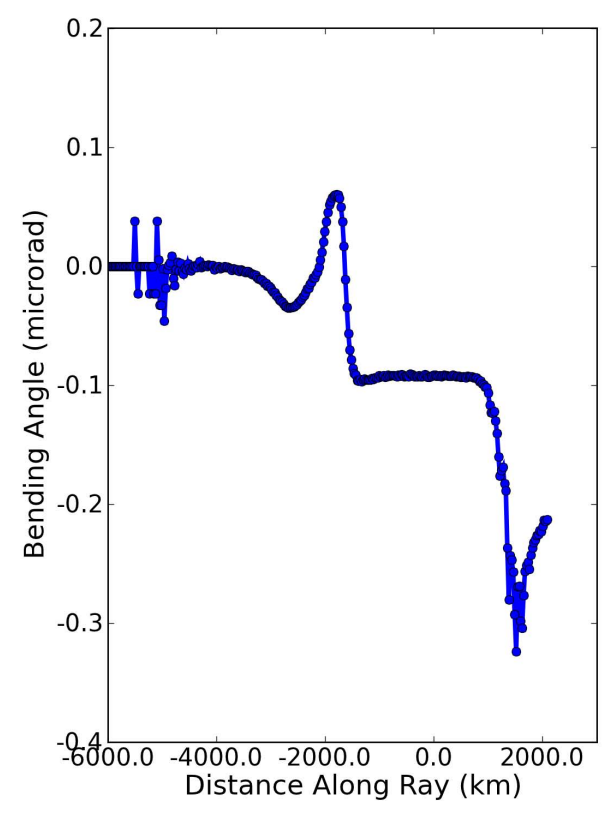

Fig. 8. Residual bending angle after the dual-frequency correction (Eq. 1) is applied, GAIM case. Compare to Fig. 5.

Residual bending angle after correction for the storm case is shown in Fig. 8. The residual follows a similar functional form for the IRI case. The bending angle affecting the retrieval is the value at the receiver location at the ray end-point. This final value is significantly larger in the GAIM case $\left(\sim-2.1 \times 10^{-7} \mathrm{rad}\right)$, compared to IRI $\left(\sim-1.5 \times 10^{-7} \mathrm{rad}\right)$. Electron density gradients associated

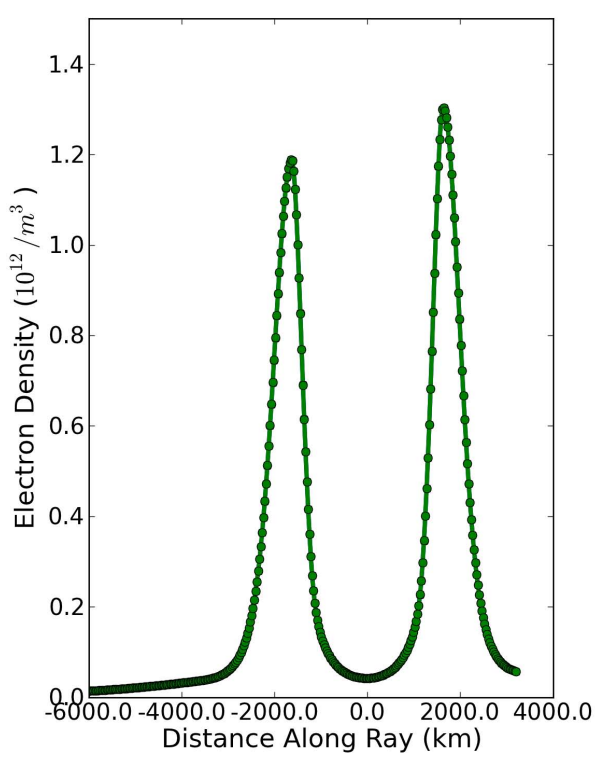

Fig. 9. Electron density along an occultation ray-path for the storm day, GAIM model case, assuming a spacecraft at COSMIC altitude of $780 \mathrm{~km}$.

with the storm-time redistribution of plasma are a likely contributing factor to the increased residual in addition to the overall scalar increase in electron density associated with the storm. Rapid fluctuations in the residual (near $-5000 \mathrm{~km}$ and $1500 \mathrm{~km}$ ) are due to numerical noise that arises when spatial refractive index derivatives are computed from the GAIM electron density grid.

\subsection{Spacecraft orbit considerations}

The orbital altitude of the GPS receiver is a significant factor determining the magnitude of the ionospheric residual. The analysis of the previous section shows that ionospheric bending in the topside ionosphere is of opposite sense to bending in the bottomside, leading to partial cancellation. The degree of cancellation depends on spacecraft altitude. The analysis just concluded is performed for a spacecraft at the CHAMP altitude of $400 \mathrm{~km}$. We have also computed residual ionospheric effects for a LEO at $780 \mathrm{~km}$ altitude, corresponding to the final altitude of the COSMIC satellites.

Figure 9 shows the electron density along an occultation ray-path for the storm day, IRI case, assuming a spacecraft at COSMIC altitude of $780 \mathrm{~km}$ (versus $400 \mathrm{~km}$ for CHAMP). The COSMIC raypath tangent point is at approximately the same location as CHAMP (within 1 degree), but the orientation is $+233^{\circ}$ with respect to North. For CHAMP, the orientation angle is $+137^{\circ}$. Orientation angle affects the electron density gradients encountered along the raypath.

The COSMIC altitude is significantly above the altitude of peak electron density $(\sim 400 \mathrm{~km})$. The electron density traces for the first and second lobes now show similar structure. For 


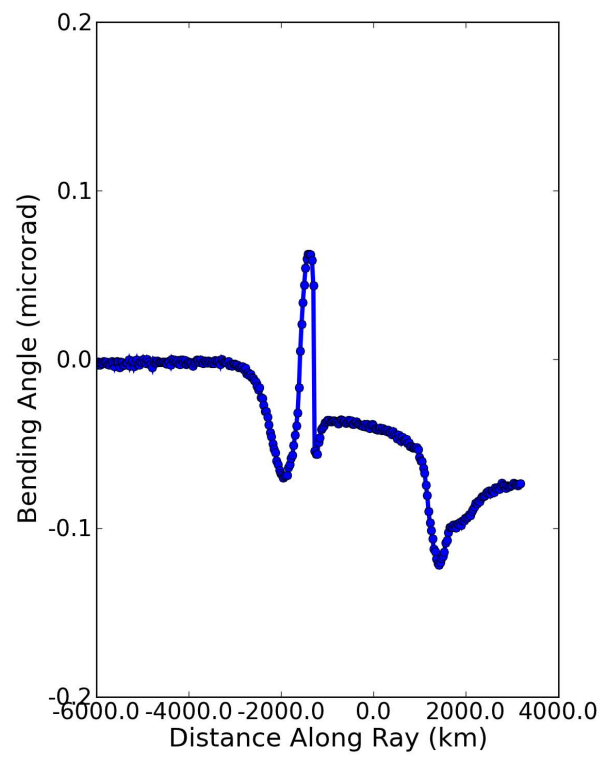

Fig. 10. Residual bending angle after the dual-frequency correction is applied, GAIM case, for a spacecraft at COSMIC altitude.

both lobes, the electron density is approximately symmetric about the peak. Bending cancellation will be more complete between bottomside and topside. In the comparable CHAMP case (Fig. 2), the electron density at the receiver is close to the peak value at the second lobe. Bending that occurs on the bottomside is not cancelled by bending on the topside. The resulting ionospheric residual bending for the COSMIC case ( $780 \mathrm{~km}$ altitude) is shown in Fig. 10. The residual bending at the spacecraft location in the COSMIC case $\left(\sim-7 \times 10^{-8} \mathrm{rad}\right)$ is approximately half the CHAMP value $\left(\sim-1.5 \times 10^{-7} \mathrm{rad}\right)$. More significantly, residual bending angle in the COSMIC case is clearly reduced by the higher orbit altitude compared to CHAMP. In the CHAMP case, the residual bending angle at the receiver location is $91 \%$ of its peak value (Fig. 2), versus only $60 \%$ of the peak value in the COSMIC case (Fig. 10). If the COSMIC receiver were at CHAMP altitude (2089 $\mathrm{km}$ distance along ray), the reduction in residual compared to the peak would be $77 \%$.

\subsection{Retrieval error}

The case studies in Sects. 4.1-4.3 illustrate the detailed dependence of ionospheric residual on large-scale ionospheric structure and raypath geometry. In this section, we perform an end-to-end simulation to calculate the error in refractivity retrieval due to the ionospheric residual. We perform a full ray-trace calculation through both the ionosphere and atmosphere and generate simulated data for use in the retrieval algorithm, applying the bending angle correction as described in Eqs. (1) and (2).

The end-to-end simulation system is diagrammed in Fig. 11. Ray-tracing is performed separately for the L1 and L2 signal paths that are propagated to a simulated receiver location at $400 \mathrm{~km}$ (CHAMP) or $780 \mathrm{~km}$ altitude (COSMIC). As described above, the IRI or GAIM electron density models were used for the ionospheric ray-tracing calculation. A spherically-symmetric (radial dependence only) refractivity profile from ECMWF analysis was used for the atmospheric ray-trace calculation, representative of conditions on 31 October 2003 near the occultation tangent point at 00:00 UTC (the exact atmospheric profile used is not relevant to the analysis). The standard retrieval process is then performed on L1 and L2 phases after perfect subtraction of geometrical distances and assuming perfectly known clocks. After calculation of bending angle at each frequency, the standard ionospheric dual-frequency correction is applied to the bending angles interpolated to common impact parameter (Eq. 1). The retrieved atmospheric refractivity is differenced with the input refractivity. The net result is an estimate of the refractivity residual resulting from imperfectly calibrated ionosphere. We restrict our study to altitudes greater than $20 \mathrm{~km}$, since below that altitude the retrieval bias due to the ionosphere decreases rapidly.

Simulation results are shown in Fig. 12 (left panel), for the GAIM and IRI cases corresponding to 30 October 2003. The raypath geometry is the same as used for the CHAMP case studies. The reason for altitude growth in refractivity bias is that the ionospheric residual remains relatively constant with altitude, whereas the atmospheric signal decreases rapidly with altitude. The net result is larger retrieval bias due to the ionospheric residual as altitude increases. Random error also increases with altitude, an effect which is not included in this simulation.

Fractional refractivity errors between $20-40 \mathrm{~km}$ altitudes are significantly larger for the GAIM (storm) case compared to IRI, as expected. The temperature errors resulting from these refractivity biases are not directly available because they depend on additional factors such as the pressure used to initialize the hydrostatic integral. As a general rule, temperature errors scale with refractivity error using a factor of $\sim 200$ (e.g. Fig. 9 of Kuo et al., 2004). Thus, the storm time refractivity biases due to residual ionosphere will produce temperature errors of approximately $0.3 \mathrm{~K}$ at $30 \mathrm{~km}$ altitude $(0.12 \%$ refractivity error), whereas during quiet time temperature errors will be closer to $0.1 \mathrm{~K}$. The storm-time errors are unacceptably large for climate trend research at altitudes of $30 \mathrm{~km}$ or higher. Kursinski et al. (1997) reported $0.12 \%$ refractivity error due to residual ionosphere at $30 \mathrm{~km}$ altitude for quiet-time solar maximum daytime conditions, versus $0.05 \%$ we find using IRI. Kursinski et al. (1997) used one-dimensional raytracing through a Chapman layer rather than the higher-fidelity IRI electron density model. These different results may be consistent with the fact that different ionospheric electron density models are used and the sensitivity to electron density vertical gradients.

Care is exercised in our retrieval simulations to ensure that ionospheric residual is the dominant error source. Another 


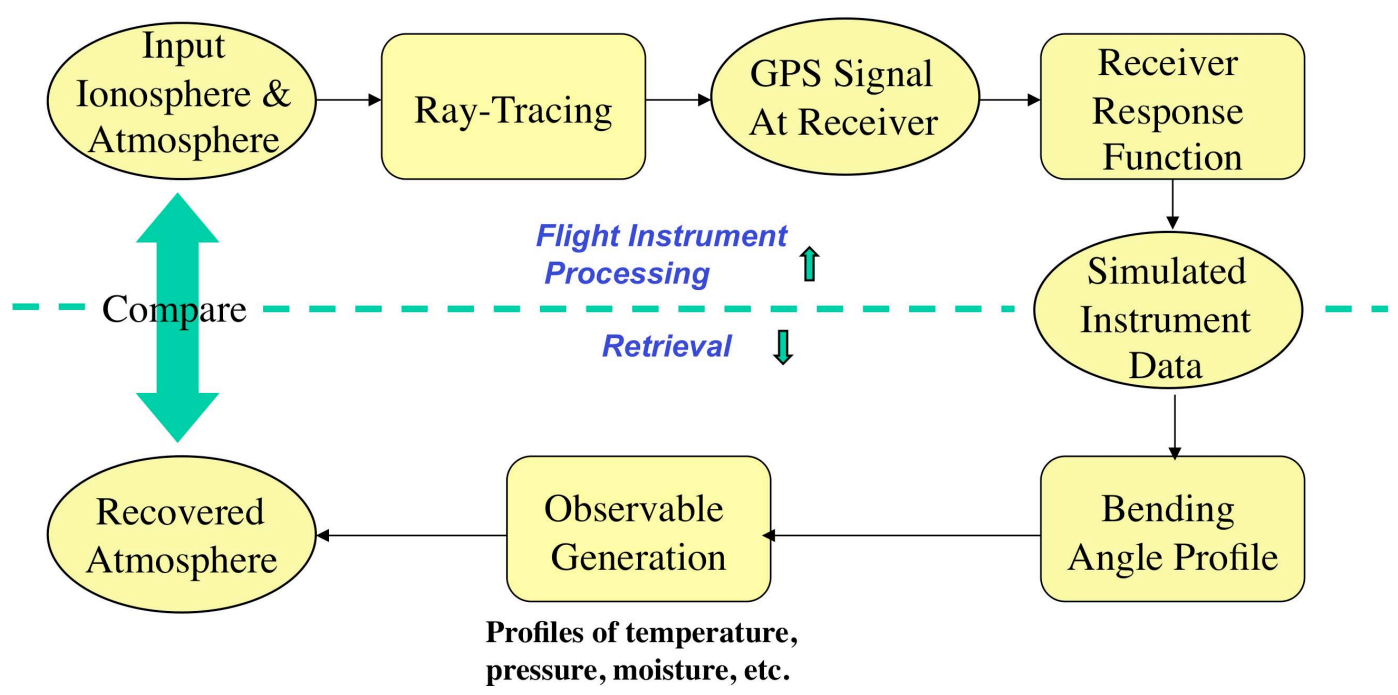

Fig. 11. Processing chain for the end-to-end simulation.

major error source that affects retrieval accuracy at high altitude is initialization of the Abel transform used to compute refractivity from bending angle (Hajj et al., 2002). The upper limit for the Abel transform is formally infinite. Often, an approximate functional form for the bending angle vertical profile is used (e.g. exponential decay) to extend the integral in altitude. Alternatively, climatological models of the neutral atmosphere might be used to compute bending angles at high altitudes. Both of these methods of extending the Abel transform beyond the altitude of the highest bending angle measurement introduce error in the retrieved refractivity profile.

To avoid retrieval error due to extrapolation, the following procedure is followed here. The retrieved refractivity profile is based on bending angle profiles derived from the raytrace calculation through the appropriate ionosphere (GAIM or IRI). This computation of refractivity requires the Abel transform, which we truncate at $70 \mathrm{~km}$ altitude rather than "infinity". This truncation introduces some error in the retrieval that is not due to ionosphere. To compensate, the "truth" refractivity we use contains the same truncation error, so that truncation error does not contribute to the differences shown in Fig. 12. The modified truth refractivity is derived, via Abel, from a "truth" bending angle profile. The truth bending angle profile is then truncated (set to zero) above $70 \mathrm{~km}$ altitude prior to the Abel being applied. (This truncation is similar to what is performed for bending angles from the raytrace simulation). The modified truth bending angle is computed exactly from the input truth refractivity (ECMWF) via the inverse Abel transform extended to $100 \mathrm{~km}$ altitude. Therefore, truncating the truth bending angle at $70 \mathrm{~km}$ and then applying the Abel transform guarantees that the modified truth refractivity and the refractivity from raytracing contain the same Abel truncation errors.
It is useful to consider the impact of electron density variation on the retrieval accuracy. The right panel of Fig. 12 shows the electron density profiles near the occultation tangent point $\left(33^{\circ} \mathrm{N}, 97^{\circ} \mathrm{W}\right)$ for the GAIM and IRI cases. Storms are not the only cause of electron density variation in the ionosphere. Variable solar insolation (X-ray and extreme ultraviolet wavelengths) and local "ionospheric weather" factors modulate electron densities during nominal conditions also. Such modulation can reach factors of two even for a common local time (Brown et al., 1991). Natural ionospheric variability and its representation in simulations may be affecting the results of different simulations appearing in the literature (e.g. Kursinski et al., 1997; Steiner et al., 2001). Refractivity bias due to ionospheric residual will generally increase with increasing electron density, of which storms can provide an extreme case (right panel, Fig. 12).

The ionospheric storm of 30 October 2003 is an extreme case producing large electron density gradients and magnitudes during daytime at particular times and locations. Such large storms typically occur only a few times per 11-yr solar cycle (generally during the declining phase of the cycle). It is instructive to consider their impact on the retrieval although such large storms do not pose practical problems for radio occultation measurements used for climate monitoring. The presence of large storms is easily detected and removed from climate averages with negligible effect. This study suggests that monitoring space weather conditions is important and that during severely disturbed conditions, atmospheric variables from RO such as temperature and pressure should be excluded from climatological averages. We emphasize that these comments apply to large-scale ionospheric structure. The impact of E-region electron density gradients is likely more significant during quiet conditions that are present the majority of the time. 

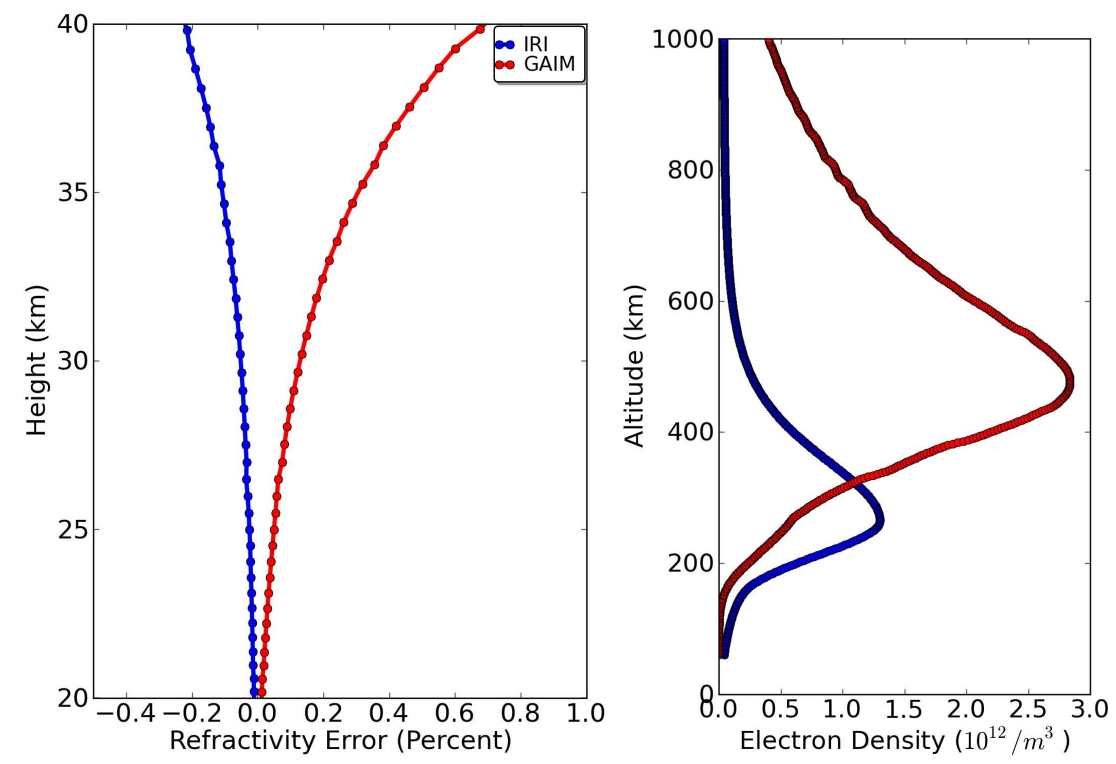

Fig. 12. Left panel: retrieval simulation results plotted as refractivity fractional error, for the IRI and GAIM cases. CHAMP satellite geometry is assumed. Right panel: vertical electron density profiles near the occultation tangent point.
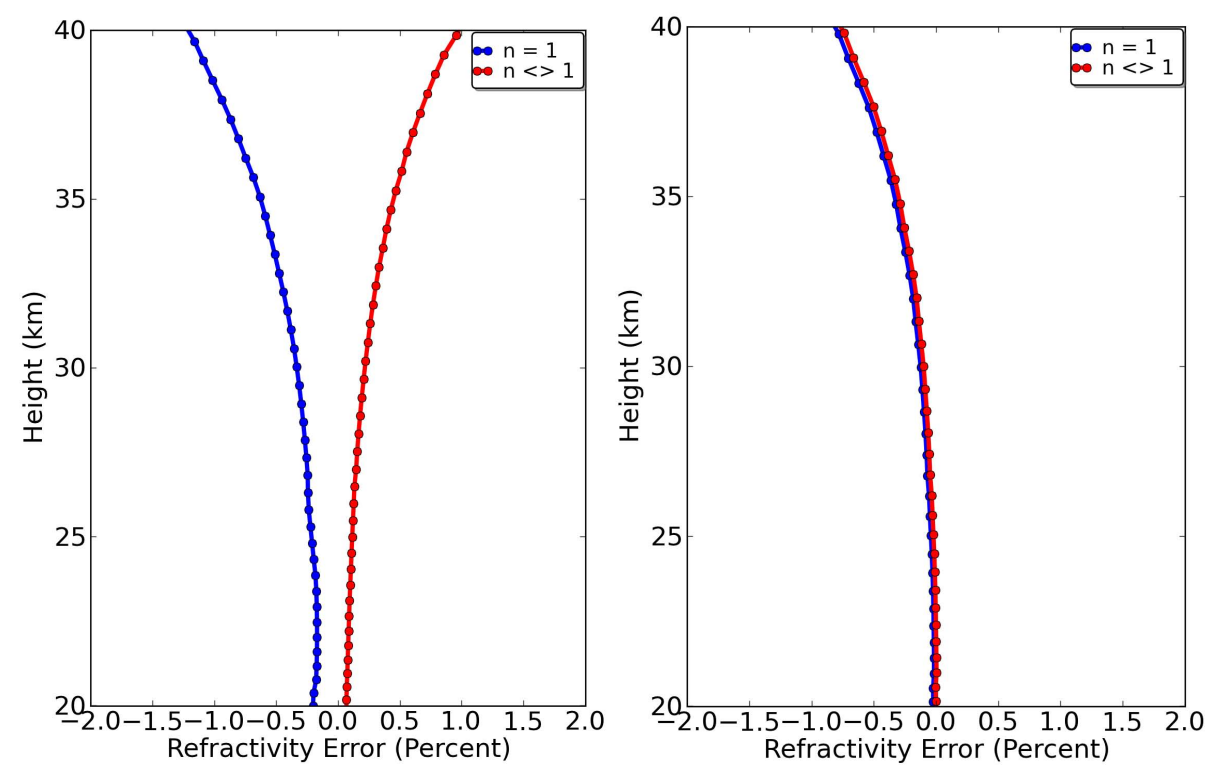

Fig. 13. Left panel: refractivity error assuming unity refractive index at the receiver, versus using the actual refractivity given by the GAIM electron density estimate, for the CHAMP raypath geometry. Right panel: same as the left panel but for the COSMIC raypath geometry.

Retrieval error is also affected by an assumption made that the refractive indices at the receiver and transmitter are both unity (Hajj et al., 2002). While unity holds at the transmitter, refractive index is not unity at the receiver, particularly when the receiver is near the peak ionospheric electron density, as is the case for CHAMP. Figure 13 shows the contribution to retrieval error from the assumption that refractive index is unity at the receiver, for the GAIM case. (The contribution to retrieval error from truncation of the Abel is also included here, unlike in Fig. 12). The left panel shows the CHAMP case, whereas the right panel shows the case for COSMIC. The retrieval difference is very significant for the CHAMP case, but negligible for COSMIC. Therefore, satellites that orbit near the peak electron density such as CHAMP are more susceptible to residual ionospheric error for two reasons: the lack of topside/bottomside cancellation discussed in Sect. 4.3, and the assumption that refractive index is unity at the receiver. Clearly, retrieval methods that 
reduce the impact of ionospheric residual need to account for refractive index at the receiver, at least for receivers near the topside ionosphere.

\section{Discussion}

This analysis shows that details of the electron density distribution and orbit altitude are two major factors in determining biases that occur due to ionospheric residual, affecting upper troposphere and lower stratosphere atmospheric retrievals. Ionospheric residual is sensitive to spacecraft altitude since the vertical distribution of ionospheric electron density can reach a peak near orbital altitudes of low Earth orbiting receivers. Residual error accumulated as rays traverse the bottom side ionosphere below the peak density tend to cancel residual error of opposite sign as the ray traverses the topside above the peak. For spacecraft near the peak electron density altitude, such as CHAMP $(\sim 400 \mathrm{~km})$, there is minimal topside/bottomside cancellation. Satellites orbiting near the peak of electron density are also more susceptible to the error made by ignoring refractive index at the receiver.

We have for the first time used an ionospheric data assimilation model to assess ionospheric residual during storm conditions. As expected, residuals increase significantly for conditions characteristic of the major disturbance known as the "Halloween Storms" of 29-30 October 2003. For long-term climate applications of GPS data, it is important to remove such active periods from climatological averages. The number of such excised days is likely to be insignificant if only the most extreme events are considered, which typically persist 1-2 days and occur perhaps 5-10 times per 11-yr solar cycle. Further research is needed to understand how the full spectrum of ionospheric disturbances can affect the residual error during the declining phase of the solar cycle, since it is likely that moderately disturbed conditions have an impact also. We note that recent solar-terrestrial research shows that during the declining phase of the solar cycle moderately active conditions can persist for days to weeks. These longduration, mild geomagnetic conditions are due to the presence of coronal holes, which migrate to lower latitudes on the solar surface during the declining phase (Tsurutani et al., 2006).

This study generally agrees with past efforts in characterizing the magnitude of the ionospheric residual on retrieval error. However, there is a spread in past research likely due to the detailed assumptions used regarding ionospheric structure, the magnitudes of electron density considered, and whether E-region layers are included. We believe that even at $20 \mathrm{~km}$ altitude, ionospheric residual remains too large for climate monitoring applications during daytime solar maximum conditions. Although past studies may correctly conclude that RO is ready for observing long-term climate (Steiner et al., 2001; Steiner et al., 2009), we believe "the margin for error" is too narrow and should be safer.
Continuing efforts are encouraged to develop algorithms that reduce the ionospheric residual error using improved algorithms and techniques (Ladreiter and Kirchengast, 1996; Gorbunov et al., 1996; Syndergaard, 2000). As discussed by Gorbunov et al. (1996) the bending angle correction formula (Eq. 1) implicitly relies on a linear relationship between bending angle and phase delay due to the ionosphere. Such linearity is violated by raypath separation and non-spherical symmetry of large-scale ionospheric structure. Clearly there are opportunities for robust correction algorithms that improve upon Eq. (1).

We emphasize that this study is restricted to ionospheric residuals due to large-scale structure. Recent work (Zeng and Sokolovskiy, 2010) has emphasized the impact of small-scale structure in the E-region ( $\sim 120 \mathrm{~km}$ altitude). Such structures are not part of the current study since neither the IRI nor GAIM models reproduce them effectively. Fortunately, for climate applications the presence of these structures generally produces distinct fluctuations in the data, so that mitigating approaches can be devised. Further work is needed to characterize the frequency and distribution of such structures in the context of global climate monitoring. We note also that at high altitudes, initialization of the Abel integral may also introduce significant errors in the retrieval (Hajj et al., 2002). These errors are likely to have a different impact than ionosphere on climatological averages formed from RO data.

It is likely that long-term climate records will combine a mix of RO satellites orbiting at varying altitudes. Our analysis shows a significant impact of orbit altitude on the magnitude of ionospheric residual bending. Therefore, care must be exercised when creating the long-term record, to avoid small systematic biases that might vary with mission. Clearly, this matter is tied to the overall question of reducing ionospheric residuals to achieve greater margin for error in forming climate averages from RO data. As part of this margin, we recommend that ionospheric activity indices be consulted to make sure that increased ionospheric activity is not affecting the record.

\section{Conclusions}

We have performed a detailed propagation study for GPS signals in an occulting geometry to gain insight into the sources of residual ionospheric bias affecting upper troposphere/lower stratospheric retrievals. This is the first study to address the case of severe geomagnetic storms that create large electron density magnitudes and spatial gradients in the ionosphere. The large resulting retrieval bias suggests that monitoring ionospheric conditions is a necessary prerequisite for long-term climate observation with RO, to excise those periods from the record where the level of ionospheric disturbance is unacceptably high.

We find also that orbit altitude affects the bias, potentially in a significant amount (inter-satellite differences could 
easily exceed $25 \%$ or more, depending on details of the orbit altitude and altitude of peak ionospheric refraction index). Care must be exercised to account for possible bias differences when forming long-term climate averages using multiple satellite time series of data.

The way forward for climate monitoring applications is to develop a strategy for setting robust upper bounds to ionospheric residual bias under a wide variety of solar and geomagnetic conditions. This upper bound is the means by which RO retrievals can maintain SI-traceability in the presence of ionospheric effects. Given the size of the bias above $25 \mathrm{~km}$, it is highly desirable to develop ionospheric correction algorithms that are more accurate and robust than the standard dual-frequency bending angle correction. Even with improved algorithms, there will be disturbances for which the residual bias is unacceptably large and in such cases the $\mathrm{RO}$ retrievals should not be included in long-term climate averages. This implies that some form of space weather monitoring should be implemented as part of the climate observation strategy, to ensure that disturbed conditions do not play a disproportionately large role in the climate averages.

Acknowledgements. The research for this paper was performed at the Jet Propulsion Laboratory, California Institute of Technology under contract with NASA. The authors wish to acknowledge support of the NASA Earth Science Directorate.

Edited by: U. Foelsche

\section{References}

Bassiri, B. and Hajj, G.: Higher-order ionospheric effects on the global positioning system observables and means of modeling them, Manuscripta Geodaetica, 18, 280-289, 1993.

Bilitza, D. and Reinisch, B. W.: International Reference Ionosphere 2007: Improvements and new parameters, Adv. Space Res., 42, 599-609, doi:10.1016/j.asr.2007.07.048, 2008.

Born, M. and Wolf, E.: Principles of Optics: Electromagnetic Theory of Propagation, Interference and Diffraction of Light, 6th Edition, Pergamon, Elmsford, NY, 1980.

Brown, L. D., Daniell, R. E., Fox, M. W., Klobuchar, J. A., and Doherty, P. H.: Evaluation of 6 ionospheric models as predictors of total electron-content, Radio Science, 26, 1007-1015, 1991.

Budden, K. G.: The Propagation of Radio Waves, Cambridge University Press, New York, NY, 1985.

Davies, K.: Ionospheric Radio, The Institution of Engineering and Technology, London, 1990.

Fox, N., Kaiser-Weiss, A., Schmutz, W., Thome, K., Young, D., Wielicki, B., Winkler, R., and Woolliams, E.: Accurate radiometry from space: an essential tool for climate studies, Philos. Trans. R. Soc. A-Math. Phys. Eng. Sci., 369, 4028-4063, doi:10.1098/rsta.2011.0246, 2011.

Goody, R., Anderson, J., and North, G.: Testing climate models: An approach, B. Am. Meteorol. Soc., 79, 2541-2549, 1998.

Gorbunov, M. E., Sokolovsky, S. V., and Bengtsson. L.: Space refractive tomography of the atmosphere: Modeling of direct and inverse problems, Rep. Max Planck Inst for Meteorol., No. 21059, Hamburg, Germany, 1996.

Hajj, G. A., Kursinski, E., R., Romans, L. J., Bertiger, W. I., and Leroy, S. S.: A technical description of atmospheric sounding by GPS occultation, J. Atmos. Solar-Terr. Phys., 64, 451-469, 2002.

Hajj, G. A., Ao, C. O., Iijima, B. A., Kuang, D., Kursinski, E. R., Mannucci, A. J., Meehan, T. K., Romans, L. J., de la Torre Juarez, M., and Yunck, T. P.: CHAMP and SAC-C atmospheric occultation results and intercomparisons, J. Geophys. Res., 109, D06109, doi:10.1029/2003JD003909, 2004.

Hayashi, H., Furumoto, J. I., Lin, X. N., Tsuda, T., Shoji, Y., Aoyama, Y. and Murayama, Y.: Validation of Refractivity Profiles Retrieved from FORMOSAT-3/COSMIC Radio Occultation Soundings: Preliminary Results of Statistical Comparisons Utilizing Balloon-Borne Observations, Terrestrial Atmos. Ocean. Sci., 20, 51-58, doi:10.3319/tao.2008.01.21.01(f3c), 2009.

He, W. Y., Ho, S. P., Chen, H. B., Zhou, X. J., Hunt, D., and Kuo, Y. H.: Assessment of radiosonde temperature measurements in the upper troposphere and lower stratosphere using COSMIC radio occultation data, Geophys. Res. Lett., 36, L17807, doi:10.1029/2009gl038712, 2009.

Hoque, M. M., and Jakowski, N.: Higher order ionospheric propagation effects on GPS radio occultation signals, Adv. Space Res., 46, 162-173, doi:10.1016/j.asr.2010.02.013, 2010.

International Association of Geomagnetism and Aeronomy (IAGA), D. V., Working Group 8: The 9th Generation International Geomagnetic Reference Field, Earth Planet. Space, 55, i-ii, 2003.

Kuo, Y. H., Wee, T. K., Sokolovskiy, S., Rocken, C., Schreiner, W., Hunt, D., and Anthes, R. A.: Inversion and error estimation of GPS radio occultation data, J. Meteorol. Soc. Jpn., 82, 507-531, 2004.

Kuo, Y. H., Schreiner, W. S., Wang, J., Rossiter, D. L., and Zhang, Y.: Comparison of GPS radio occultation soundings with radiosondes, Geophys. Res. Lett., 32, L05817, doi:10.1029/2004g1021443, 2005.

Kursinski, E. R., Hajj, G. A., Bertiger, W. I., Leroy, S. S., Meehan, T. K., Romans, L. J., Schofield, J. T., McCleese, D. J., Melbourne, W. G., Thornton, C. L., Yunck, T. P., Eyre, J. R., and Nagatani, R. N.: Initial results of radio occultation observations of Earth's atmosphere using the global positioning system, Science, 271, 1107-1110, 1996.

Kursinski, E. R., Hajj, G. A., Schofield, J. T., Linfield, R. P., and Hardy, K. R.: Observing Earth's atmosphere with radio occultation measurements using the Global Positioning System, J. Geophys. Res.-Atmos., 102, 23429-23465, 1997.

Ladreiter, H. P. and Kirchengast, G.: GPS/GLONASS sensing of the neutral atmosphere: Model-independent correction of ionospheric influences, Radio Science, 31, 877-891, 1996.

Mannucci, A. J., Wilson, B. D., Yuan, D. N., Ho, C. H., Lindqwister, U. J., and Runge. T. F.: A global mapping technique for GPSderived ionospheric total electron content measurements, Radio Science, 33, 565-582, 1998.

Mannucci, A. J., Tsurutani, B. T., Iijima, B. A., Komjathy, A., Saito, A., Gonzalez, W. D., Guarnieri, F. L., Kozyra, J. U., and Skoug, S.: Dayside global ionospheric response to the major interplanetary events of October 29-30, 2003 "Halloween storms", Geophys. Res. Lett., 32, L12S02, doi:10.1029/2004g1021467, 2005.

Mannucci, A. J., Ao, C. O., Yunck, T. P., Young, L. E., Hajj, 
G. A., Iijima, B. A., Kuang, D., Meehan, T. K., and Leroy, S. S.: Generating climate benchmark atmospheric soundings using GPS occultation data, SPIE Proceedings, 6301, 630108, doi:10.1117/12.683973, 2006.

Ohring, G., Wielicki, B., Spencer, R., Emery, B., and Datla, R.: Satellite instrument calibration for measuring global climate change - Report of a Workshop, B. Am. Meteorol. Soc., 86, 1303-1313, 2005.

Rocken, C., Anthes R., Exner M., Hunt, D., Sokolovskiy, S., Ware, R., Gorbunov, M., Schreiner, W., Feng, D., Herman, B., Kuo, Y. H., and Zou, X.: Analysis and validation of GPS/MET data in the neutral atmosphere, J. Geophys. Res.-Atmos., 102, 2984929866, 1997.

Schreiner, W., Rocken, C., Sokolovskiy, S., Syndergaard, S., and Hunt, D.: Estimates of the precision of GPS radio occultations from the COSMIC/FORMOSAT-3 mission, Geophys. Res. Lett., 34, L04808, doi:10.1029/2006gl027557, 2007.

Sokolovskiy, S., Schreiner, W., Rocken, C., and Hunt, D.: Optimal Noise Filtering for the Ionospheric Correction of GPS Radio Occultation Signals, J. Atmos. Ocean. Technol., 26, 1398-1403, doi:10.1175/2009jtecha1192.1, 2009.

Steiner, A. K. and Kirchengast, G.: Error analysis for GNSS radio occultation data based on ensembles of profiles from endto-end simulations, J. Geophys. Res.-Atmos., 110, D15307, doi:10.1029/2004JD005251, 2005.

Steiner, A. K., Kirchengast, G., and Ladreiter, H. P.: Inversion, error analysis, and validation of GPS/MET occultation data, Ann. Geophys.-Atmos. Hydrospheres Space Sci., 17, 122-138, 1999.

Steiner, A. K., Kirchengast, G., Foelsche, U., Kornblueh, L., Manzini, E., and Bengtsson, L.: GNSS occultation sounding for climate monitoring, Phys. Chem. Earth Pt. A-Solid Earth Geod., 26, 113-124, 2001.

Steiner, A. K., Kirchengast, G., Lackner, B. C., Pirscher, B., Borsche, M., and Foelsche, U.: Atmospheric temperature change detection with GPS radio occultation 1995 to 2008, Geophys. Res. Lett., 36, L18702, doi:10.1029/2009g1039777, 2009.
Sun, B. M., Reale, A., Seidel, D. J., and Hunt, D. C.: Comparing radiosonde and COSMIC atmospheric profile data to quantify differences among radiosonde types and the effects of imperfect collocation on comparison statistics, J. Geophys. Res.-Atmos., 115, D23104, doi:10.1029/2010jd014457, 2010.

Syndergaard, S.: On the ionosphere calibration in GPS radio occultation measurements, Radio Science, 35, 865-883, 2000.

Tsurutani, B. T., Gonzalez, W. D., Gonzalez, A. L. C., Guarnieri, F. L., Gopalswamy, N., Grande, M., Kamide, Y., Kasahara Y., Lu, G., Mann I., McPherron, R., Soraas F., and Vasyliunas, V.: Corotating solar wind streams and recurrent geomagnetic activity: A review, J. Geophys. Res-Space Phys., 111, A07S01, doi:10.1029/2005JA011273, 2006.

Vergados, P. and Pagiatakis, S. D.: First estimates of the second-order ionospheric effect on radio occultation observations, J. Geophys. Res-Space Phys., 115, A07317, doi:10.1029/2009ja015161, 2010.

Vorobev, V. V. and Krasilnikova, T. G.: An estimation of accuracy of the atmospheric refractive-index recovery from measurements of doppler shifts at frequencies used in the navstar system, Izvestiya Akademii Nauk Fizika Atmosfery I Okeana, 29, 602-609, 1993.

Wang, C. M., Hajj, G., Pi, X. Q., Rosen, I. G., and Wilson, B.: Development of the Global Assimilative Ionospheric Model, Radio Science, 39(1), RS1S06, doi:10.1029/2002rs002854, 2004.

Wickert, J., Reigber, C., Beyerle, G., König, R., Marquardt, C., Schmidt, T., Grunwaldt, L., Galas, R., Meehan, T. K., Melbourne, W. G., and Hocke, K.: Atmosphere sounding by GPS radio occultation: First results from CHAMP, Geophys. Res. Lett., 28, 3263-3266, doi:10.1029/2001g1013117, 2001.

Xu, X. H., Luo, J., and Shi, C. A.: Comparison of COSMIC Radio Occultation Refractivity Profiles with Radiosonde Measurements, Adv. Atmos. Sci., 26, 1137-1145, doi:10.1007/s00376009-8066-y, 2009.

Zeng, Z. and Sokolovskiy, S.: Effect of sporadic E clouds on GPS radio occultation signals, Geophys. Res. Lett., 37, L18817, doi:10.1029/2010g1044561, 2010. 